\title{
A hidden echocardiographic pitfall: the Gerbode defect
}

\author{
Daniela Colomba $\cdot$ Mauro Cardillo $\cdot$ \\ Alessandro Raffa $\cdot$ Christiano Argano • \\ Giuseppe Licata
}

Received: 26 August 2013/Accepted: 7 October 2013/Published online: 20 October 2013

(c) SIMI 2013

The Gerbode defect is characterized by a perimembranous ventricular septal defect (VSD) between the left ventricle and the right atrium. It is a rare defect representing less than $1 \%$ of congenital cardiac defects [1]. Acquired cases have been described, most often due to endocarditis, but also secondary to valvular surgery, thoracic trauma and ischemic heart disease. Frank Gerbode was the first surgeon to report a successful series of patients who underwent surgery for left ventricular to right atrium shunt in 1958 [2].

Gerbode has described two types of defect:

- Type A: In this form, the concurrent presence of a perimembranous VSD plus the tricuspid valve defect are visible. The shunt starts from the left ventricle to the right ventricle and through the tricuspid valve into the right atrium. This is referred to as an indirect left ventricle-right atrium shunt.

- Type B: This form is characterized by a left ventricle to the right atrium shunt.

This rare form of interventricular septal defect should be suspected during the performance of an echocardiogram, when there is an unusually dilatated right atrium, and

Electronic supplementary material The online version of this article (doi:10.1007/s11739-013-1009-8) contains supplementary material, which is available to authorized users.

D. Colomba $(\bowtie) \cdot$ M. Cardillo

Echocardiography Laboratory, Biomedical Department of Internal Medicine and Specialities (DiBiMIS), University of Palermo, Piazza delle Cliniche, 2, 90127 Palermo, Italy

e-mail: colomda@yahoo.it

A. Raffa · C. Argano - G. Licata

Biomedical Department of Internal Medicine and Specialities

(DiBiMIS), University of Palermo, 90127 Palermo, Italy when, in the presence of an interventricular septal defect, high velocity flows or aliasing are absent in the right ventricle (mainly in inflow tract) [3]. A particular feature of the Gerbode defect is the high Doppler gradient of the shunt, due to the higher pressure gap between the left ventricle and the right atrium rather than left ventricleright ventricle (especially in the presence of pulmonary hypertension).

We report the case of a 48-year-old woman admitted to our division because of mild exertional dyspnoea. Her clinical history included a previous diagnosis of a perimembranous VSD, in adolescence. Physical examination was unremarkable except for a 3/6 Levine pansystolic murmur heard at the left sternal border. The echocardiogram detected only a mild right atrium enlargement. The presence of a perimembranous ventricular septal was initially confirmed, but a careful color Doppler flow mapping analysis did not show high velocity flows in the right ventricle inflow during systole although high velocity flows were well present in the right atrium. How was it possible? Why were high velocity flows present only in the right atrium? This finding might suggest the presence of a tricuspid regurgitation and pulmonary hypertension defect (jet velocity $4.87 \mathrm{~m} / \mathrm{s}, 95 \mathrm{mmHg}$ gradient estimated). Doubts about the previous diagnosis were raised. For this reason a color Doppler analysis, frame by frame, was performed. Surprisingly, the short axis projection showed a sudden $90^{\circ}$ turn of the jet from the left ventricle to the right atrium concurrent with the closure of the septal leaflet of the tricuspid valve (Fig. 1). The jet continued its course depicting the atrial surface of the tricuspid valve. The subcostal views of the right ventricle confirmed our analysis. A small thickening and abnormal insertion of the basal portion of the septal tricuspid leaflet were also present. These images were consistent with a left to right 


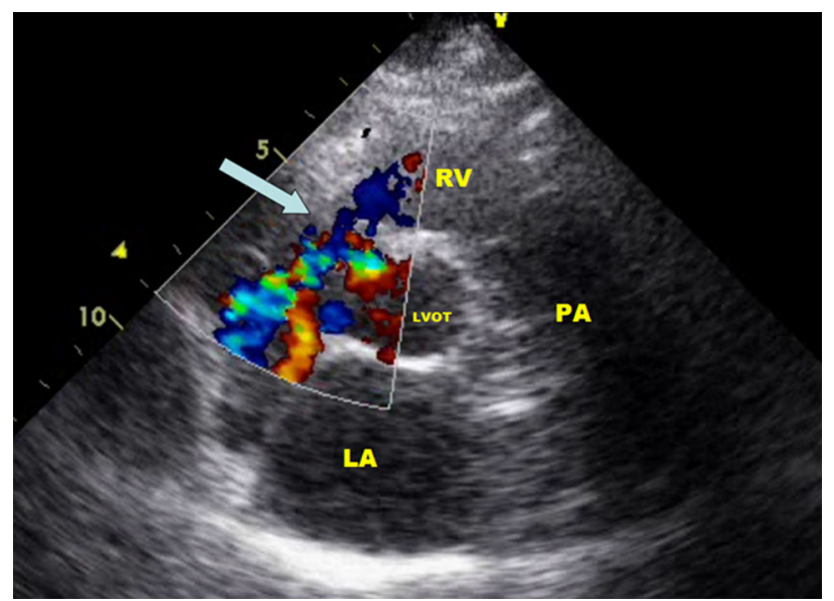

Fig. 1 Short axis view at the level of the left ventricle outflow tract: the image is recorded in early systole. Sudden turn (arrow) of the jet due to the tricuspid closure is evident. No high velocity flows are detectable in the right ventricle. $L A$ left atrium, $P A$ pulmonary artery, $R V$ right ventricle, $L V O T$ left ventricle outflow tract

atrial communication, namely Gerbode defect type A. The patient history did not reveal any previous surgical intervention of the heart valves, neither endocarditis nor acute trauma or myocardial infarction, all causes of acquired Gerbode-type defect. In view of this, our case is most likely a congenital defect.

In conclusion, a superficial glance in the technical execution of echocardiography instead of a careful and critical examination could produce a misinterpretation that might have a direct impact on clinical decision making.

Conflicts of interest We have no conflicts to disclose.

\section{References}

1. Wasserman SM, Fann JI, Atwood JE, Burdon TA, Fadel BM (2002) Acquired left ventricular-right atrial communication: Gerbode-type defect. Echocardiography 19:67-72

2. Kelle AM, Young L, Kaushal S, Duffy CE, Anderson RH, Backer CL.(2013) The Gerbode defect: a ventriculo-atrial defect. http:// www.ctsnet.org/sections/clinicalresources/congenital/article-24. html Accessed 3 Aug 2013

3. Faramarz T, Mohammad-Reza M (2007) How to prevent echocardiographic misinterpretation of Gerbode type defect as pulmonary hypertension. Eur J Echocardiogr 8:494-497 